Diabetologia, Suppl. to 9, $311-316$ (1973)

(C) by Springer-Verlag 1973

\title{
Pharmacodynamic Aspects of Two Sulphonylurea Derivatives Glipizide and Glibenclamide
}

\author{
D. Artini, R. Abbiati, G. Orsini ${ }^{1}$, M.A. Parenti, K. Bloch, S. Daturi ${ }^{2}$ and V. Mandelli ${ }^{3}$ \\ Carlo Erba Research Institute, Milan, Italy \\ Received: February 9, 1973, and in revised form: May 28, 1973, accepted: May 28, 1973
}

\begin{abstract}
Summary. The results of experiments in dogs with and without two glucose loads and in the isolated pancreas to compare the pharmacodynamics of two low-dosage arylsulphonylureas, glipizide and glibenclamide, are described and discussed. - The results agree with those obtained by other authors confirming that glibenclamide shows delayed but prolonged activity on both plasma insulin and glucose levels. Moreover glibenclamide counteracts the hyperglycaemia induced by the second glucose load less efficently. Glipizide acts faster on insulin and glucose
\end{abstract}

levels, which return quickly to normal. When a second glucose load was given it was still more active in reducing plasma glucose levels. The dynamics of insulin secretion following glipizide more closely resembles tolbutamide than glibenclamide.

Key words: Glipizide, glibenclamide; in vitro, in vivo, dynamics, of insulin secretion, dynamics of hypoglycaemic activity, effect on repeated glucose loads.
During pharmacological trials on a series of pyrazinoyl-amino-ethyl-benzene-sulphonylureas with oral hypoglycaemic activity $[1,2,3,4]$ the reference substance used was glibenclamide, a low dosage sulphonylurea.

Although the new molecules showed approximately the same degree of hypoglycaemic activity as glibenclamide, the blood insulin and glucose time courses differed, and it did not seem adequate to interpret the differences merely in the light of pharmacokinetics and bioavailability $[5,6]$.

This paper illustrates some pharmacodynamic aspects of one compound in this series, glipizide or K 4024 (N-\{4-[beta-(5-methyl-pyrazine - 2 -carboxyamido)ethyl - benzene-sulphonyl $\}-N$ 'cyclohexyl - urea), compared with glibenclamide.

\section{Material and Methods}

The first experiment was planned to check the plasma glucose and insulin pattern following oral administration of glipizide or glibenclamide in the dog. Twelve Beagle dogs from our stock were assigned at random to the two treatments. The dogs were fasted for $16 \mathrm{~h}$. The products, micronised and suspended in $0.5 \%$ Methocel, were administered by gavage at the dose of $120 \mathrm{\gamma} / \mathrm{kg}$. Blood samples were taken at time 0 (basal), and $20,30,40,60,90,120$ and $180 \mathrm{~min}$ following administration of the test products.

Glucose and insulin assay was carried out on plasma obtained by centrifuging immediately after taking the sample. The direct o-toluidine method of Frings et al. [7] was used for glucose assay; insulin

\footnotetext{
1 Cytopharmacology Laboratory;

2 Pharmacology Laboratory;

3 Biometry Centre.
}

was determined by the radio-immunological double antibody method of Hales and Randle [8], using the insulin immunoassay kit from the Amersham Radiochemical Centre.

Statistical analysis was carried out on the results of this trial to show up the following four aspects:

1. comparison of time courses: analysis of variance was carried out using three classification criteria (treatments, dogs, times). The least significant difference for $p=0.05$ and $p=0.01$ was also calculated to compare the means for the two drugs;

2 . comparison of maximum peaks : for each animal the maximum peak was noted and the means for the two drugs were compared by analysis of variance;

3. comparison of maximum peak times: the time required to reach maximum peak was noted for each animal, and these times were transformed into reciprocals, then compared by analysis of variance. The harmonic mean of the times was also calculated;

4. comparison of the areas underneath the plasma glucose and insulin time course curves: the area was calculated for each dog, and the means of the areas were compared by analysis of variance.

The second experiment was set up to compare the effects of glipizide and glibenclamide on hyperglycaemia and hyperinsulinaemia induced by two glucose loads in the dog. Twelve Beagles were used, acting first as controls; they were then randomly divided in two groups of 6 ; one was given glipizide and the other glibenclamide. A blood sample was taken, and basal values (time 0 ) determined. The animals then received either the vehicle alone by gavage (controls) or else the glipizide or glibenclamide suspension in $0.5 \%$ Methocel at the dose of $120 \mathrm{\gamma} / \mathrm{kg}$; immediately after taking the 40 and 420 min samples, the animals were given $4 \mathrm{ml} / \mathrm{kg}$ of a $33 \%$ glucose solution, by gavage. Plasma glucose and insulin levels were followed every $20 \mathrm{~min}$ for $100 \mathrm{~min}$. 
Glucose and insulin assay methods were as described in the first experiment.

In order to evaluate the significance of the differences between the plasma glucose and insulin means at the different times for the three treatments, Kramer's test [9] was used, since the three samples involved different numbers. Further statistical analysis was made as described earlier.

Another series of experiments was carried out on isolated, perfused rat pancreas using the method described by Loubatières [10].

Outbred SPF CFE male rats from our stock, weighing $350-450 \mathrm{~g}$, were used; access to food and water was $a d l i b$. Anaesthesia was induced by intraperitoneal injection of ethyl urethane $(1 \mathrm{~g} / \mathrm{kg})$, and respiration maintained throughout the experiment with a mixture of $\mathrm{O}_{2^{-}} \mathrm{CO}_{2}(95-5 \%)$. The pancreas was separated from all surrounding tissue and perfused at $37^{\circ} \mathrm{C}$, using an open eircuit system. The basic perfusion medium was Krebs-Ringer solution containing $2 \%$ Grad/V bovine serum albumin (Sigma $\mathrm{Ch}$. Co.) and $0.8 \mathrm{~g} / \mathrm{l}$ glucose. The perfusion rate was approximately $2.5 \mathrm{ml} / \mathrm{min}$, pressure $20-30 \mathrm{mmHg}$. Insulin secretion stabilized in all cases in about $30 \mathrm{~min}$; from then on the test substance was added to the perfusion medium using an infusion pump, dissolved $2.5 \gamma$ in $5 \mathrm{ml}$ of the perfusion solution. Infusion time was $15 \mathrm{~min}$; while flowing through the pancreas the products reached a concentration of $50 \gamma / 1$. When stimulus with the drugs was stopped, perfusion was carried on with the basic solution.

Samples for radio-immunological insulin assay [8] were drawn from the solution as it came out of the pancreas at the times indicated in Fig. 5.

The two means at the various times were compared by a non-parametric statistical method, Wilcoxon's non-paired data test [11].

\section{Results}

\section{Plasma Glucose and Insulin in the Dog Following Oral Administration of Glipizide or Glibenclamide}

Fig. 1 gives the plasma glucose levels recorded in this test, and Fig. 2 the plasma insulin levels.

The effect of glipizide on plasma glucose starts to become evident $30 \mathrm{~min}$ after administration, reaching its maximum at $94 \mathrm{~min}$ (harmonic mean). Glibenclamide takes longer to act; its effect becomes evident only after 90 min, reaching maximum at a time which may be estimated at over $160 \mathrm{~min}$ (to obtain an exact figure the experiment would have had to be prolonged beyond $180 \mathrm{~min}$ ).

Comparison of the plasma glucose means shows a significant difference between glipizide and glibenclamide at the 90 th min.

Analysis of the plasma glucose time courses shows a highly significant interaction, times $x$ drugs, con- firming that glipizide acts faster. Comparison of the areas beneath the plasma glucose concentration curves also shows a significant difference between the two drugs.

The results in Fig. 2, regarding plasma insulin levels, confirm that glipizide acts faster than glibenclamide. Analysis of the time to peak shows a highly

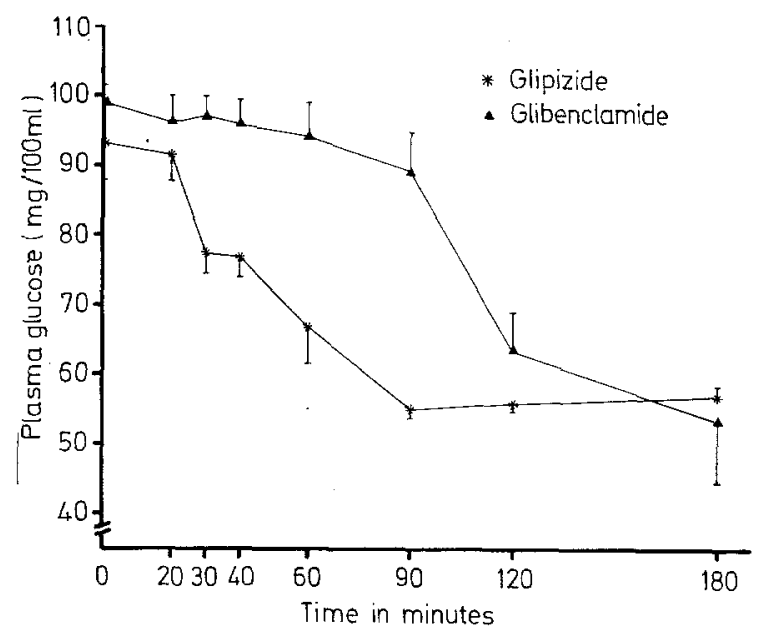

Fig. 1. Plasma glucose levels in dogs at different times after oral administration of glipizide and glibenclamide, $120 \gamma / \mathrm{kg}$

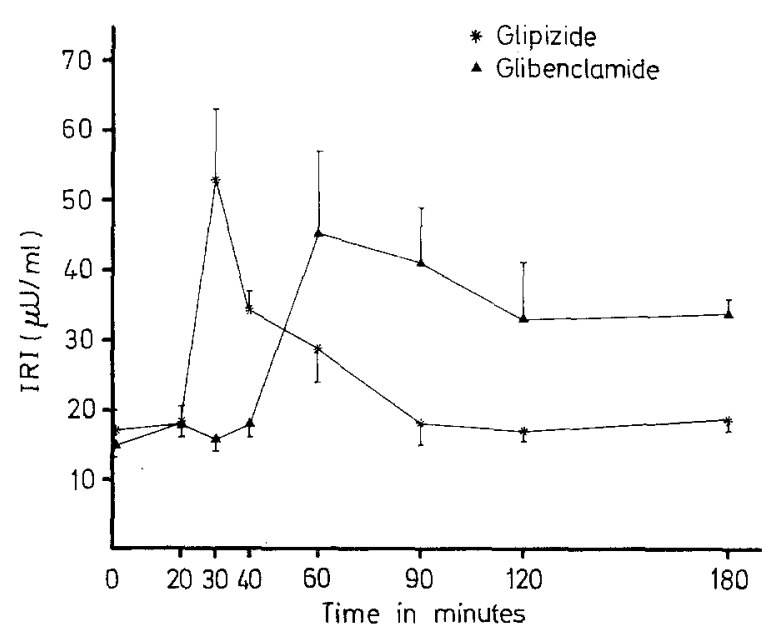

Fig. 2. Plasma insulin (IRI) levels in dogs at different times after oral administration of glipizide and glibenclamide, $120 \gamma / \mathrm{kg}$

significant difference between the two drugs; the harmonic mean was $31 \mathrm{~min}$ for glipizide and $72 \mathrm{~min}$ for glibenclamide.

The main difference between the two drugs, however, is the fact that plasma insulin remains high longer with glibenclamide.

With glipizide, following the peak the insulin level decreases rapidly to reach basal values at the 90th min, whereas with glibenclamide the decrease in 
plasma insulin is much less marked and the plasma levels become normal only after the 180th min.

From the 60th min onwards, there was a highly significant difference between the mean plasma insulin level for the two drugs. Statistical analysis confirms the difference in the plasma insulin levels induced by the two drugs; there is a highly significant interaction times $\mathrm{x}$ drugs.

In view of the variations shown by the results, the means of the areas underneath the plasma in sulin curves for the two drugs were not significantly different, although the mean for glibenclamide was $50 \%$ higher than for glipizide.

Effect of Glipizide and Glibenclamide on Plasma Glucose and Insulin (IRI) Levels Increase Induced in the Dog by Two Glucose Loads

Fig. 3 and Table 1 give the results of this experiment with reference to the plasma glucose levels, and Fig. 4 and Table 2 refer to plasma insulin levels.

In animals treated with glipizide the mean plasma glucose level at the 40th min was already significantly lower than both the controls and the glibenclamide group; this indicates that glipizide acts faster.

Throughout the first phase of the experiment (1 st glucose load) the glipizide-induced plasma glucose pattern remained lower than with the other two treatments; the means were significantly lower up to the 120th min. At the 140th min the means for the two hypoglycaemic compounds were not significantly different, although they differed significantly from the controls. The plasma glucose levels induced by glibenclamide start to differ from the controls only after the 60 th min.

Seven hours after treatment, the mean plasma glucose level induced by glipizide was not significantly different from the controls, whereas the glibenclamidetreated animals showed a significantly lower mean than both the controls and those treated with glipizide.

During the second phase of the experiment, following the second glucose load, plasma glucose levels rose more in the animals treated with glibenclamide and the controls than in the group treated with glipizide. The effect of glibenclamide became evident only at the 480 th min, and appeared more marked; in fact at the 500th min the glibenclamide-induced plasma glucose levels were significantly lower than in the other two groups.

Glipizide thus appears to act faster and less drastically, considering that following the stimulus represented by the hyperglycaemic load, the product lowered plasma glucose to within physiological limits, fairly close to control levels.

The figure giving the plasma insulin level time courses (Fig. 4) and the results in Table 2, show that at the 40th min the two groups treated with hypoglycaemic compounds showed higher plasma insulin levels than controls. However, in actual fact only the group receiving glipizide showed a real increase, since by chance the mean basal plasma insulin level in the glibenclamide group was higher than in the other two groups.

At 60th min glipizide also induced higher plasma insulin levels, with significantly higher means than

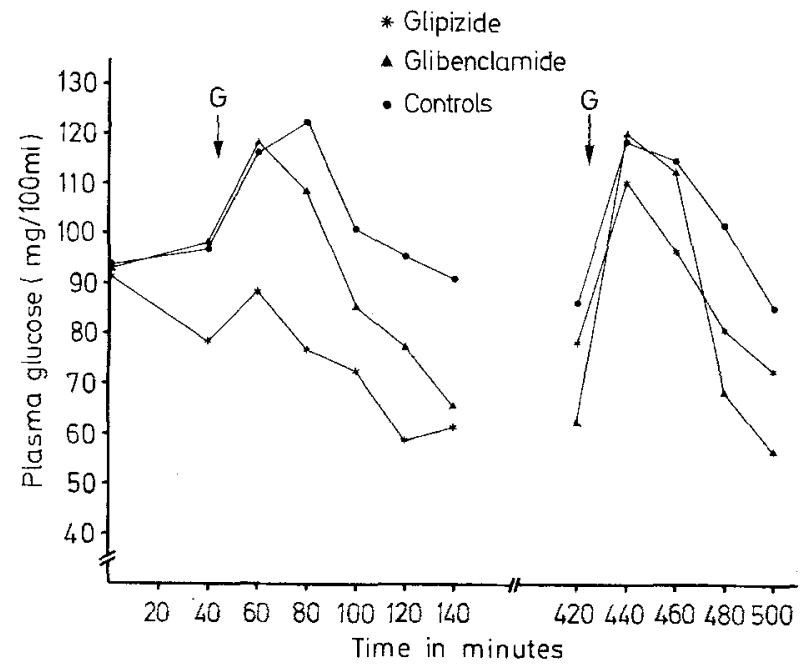

Fig. 3. Plasma glucose variations in control dogs and in dogs treated orally with glipizide or glibenclamide, $120 \gamma / \mathrm{kg}$, and given glucose loads $(G) 40$ and $420 \mathrm{~min}$ after administration (see Table 2)

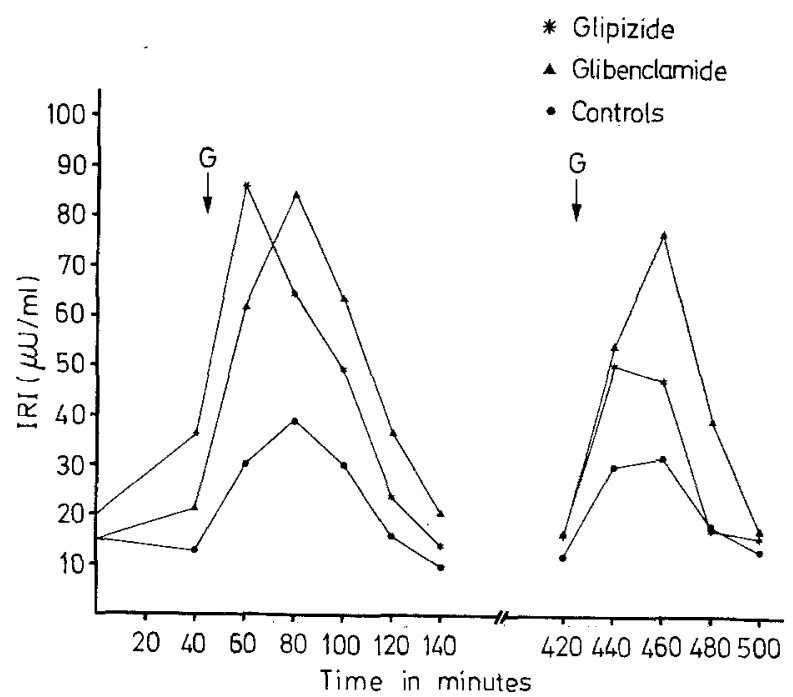

Fig. 4. Plasma insulin variations (IRI) in control dogs and in dogs treated orally with glipizide or glibenclamide, $120 \mathrm{\gamma} / \mathrm{kg}$, and given glucose loads (G) 40 and $420 \mathrm{~min}$ after administration (see Table 2)

glibenclamide. At the 80th min plasma insulin was already decreasing in the group treated with glipizide, but in the glibenclamide and control groups a further rise was noted; after this the decrease in plasma insulin was almost parallel in all groups. 
Statistical analysis of the results following the first glucose load further confirmed the difference between the two treatments. Both as regards the insulin peaks and the areas beneath the plasma insulin level curves, the means for the two hypoglycaemic agents are not significantly different, but they do differ significantly from the controls. A significant difference was found between the two drugs as regards the times required to reach peak. The harmonic means
The three harmonic means for the peak times show no significant differences although the glibenclamide mean was delayed $7 \mathrm{~min}$ as compared to glipizide.

\section{Insulin Secretion by the Isolated, Perfused Rat Pancreas Induced by Glipizide and Glibenclamide}

Fig. 5 shows that at the start of perfusion with the two products, the pancreas responded rapidly

Table 1. Means, standard errors and results of comparisons of treatments for plasma glucose-levels obtained at different times in control dogs and dogs treated orally with glipizide or glibenclamide, $120 \mathrm{\gamma} / \mathrm{kg}$, and given 2 oral glucose loads 40 and 420 min after drug administration

\begin{tabular}{|c|c|c|c|c|c|c|}
\hline \multirow{2}{*}{$\begin{array}{l}\text { Time } \\
\text { in minutes }\end{array}$} & \multicolumn{3}{|c|}{ Plasma glucose $(\mathrm{mg} / 100 \mathrm{ml})$. Means \pm S.E. } & \multicolumn{3}{|c|}{ a Comparisons by Kramer's test } \\
\hline & $\begin{array}{l}\text { Controls } \\
\text { (C) no. }=12\end{array}$ & $\begin{array}{l}\text { Glipizide } \\
(G Z) \text { no. }=6\end{array}$ & $\begin{array}{l}\text { Glibenclamide } \\
\text { (GC) no. }=6\end{array}$ & C vs GZ & $\mathrm{C}$ vs GC & GC vs GZ \\
\hline 0 & $93.75 \pm 2.06$ & $91.33 \pm 3.50$ & $93.50 \pm 3.49$ & N.S. & N.S. & N.S. \\
\hline 40 & $96.75-2.56$ & $78.33+2.72$ & $98.17+3.47$ & H.S. & N.S. & H.S \\
\hline 60 & $116.17-2.45$ & $88.33 \pm 3.21$ & $118.33 \pm 4.98$ & H.S. & N.S. & H.S. \\
\hline 80 & $122.25 \pm 2.88$ & $76.50 \pm 4.22$ & $108.33 \pm 3.89$ & H.S. & & H.S. \\
\hline 100 & $100.58 \pm 2.63$ & $72.33 \pm 3.79$ & $85.17 \pm 2.66$ & H.S. & H.S. & $\mathrm{S}$ \\
\hline 120 & $95.33-2.44$ & $58.50 \pm 5.16$ & $77.17 \pm 2.69$ & H.S. & H.S. & H.S. \\
\hline 140 & $90.75 \pm 2.26$ & $61.33 \pm 3.87$ & $65.33 \pm 2.19$ & H.S. & H.S. & N.S. \\
\hline 420 & $86.08-2.09$ & $78.00 \pm 3.41$ & $62.00 \pm 3.78$ & N.S. & H.S. & H.S. \\
\hline 440 & $118.58 \pm 2.03$ & $110.33 \pm 3.95$ & $120.17 \pm \mathbf{3 . 4 7}$ & N.S. & N.S. & $\mathrm{S}$ \\
\hline 460 & $114.50 \pm 1.34$ & $96.33 \pm 3.98$ & $112.33 \pm 2.40$ & H.S. & N.S. & H.S. \\
\hline 480 & $101.33 \pm 1.95$ & $80.50 \pm 2.75$ & $68.17 \pm 2.66$ & H.S. & H.S. & H.S. \\
\hline 500 & $85.00 \pm 1.86$ & $72.33+2.50$ & $56.33 \pm 2.51$ & H.S. & H.S. & H.S. \\
\hline
\end{tabular}

a N.S. $=$ Not Significant $(P>0.05)$

$\mathrm{S}=$ Significant $(0.01<P \leqslant 0.05)$

H.S. $=$ Highly significant $(P \leqslant 0.01)$

Table 2. Means, standard errors and results of comparisons of treatments for plasma insulin-levels obtained at different times in control dogs and dogs treated orally with glipizide or glibenclamide, $120 \mathrm{\gamma} / \mathrm{kg}$, and given 2 oral glucose loads 40 and 420 min after drug administration

\begin{tabular}{|c|c|c|c|c|c|c|}
\hline \multirow{2}{*}{$\begin{array}{l}\text { Time } \\
\text { in minutes }\end{array}$} & \multicolumn{3}{|c|}{ IRI $(\mu \mathrm{U} / \mathrm{ml})$. Means \pm S.E. } & \multicolumn{3}{|c|}{ a Comparisons by Kramer's test } \\
\hline & $\begin{array}{l}\text { Controls } \\
\text { (C) no. }=12\end{array}$ & $\begin{array}{l}\text { Glipizide } \\
\text { (GZ) no. }=6\end{array}$ & $\begin{array}{l}\text { Glibenclamide } \\
\text { (GC) no. }=6\end{array}$ & $\mathrm{C}$ vs GZ & $\mathrm{C}$ vs $\mathrm{GC}$ & GC vs GZ \\
\hline 0 & $14.42 \pm 1.29$ & $14.17 \pm 0.98$ & $20.00 \pm 2.02$ & N.S. & $\mathrm{S}$ & $\mathbf{S}$ \\
\hline 40 & $12.58 \pm 1.46$ & $36.33 \pm 2.35$ & $21.17 \pm 2.41$ & H.S. & H.S. & H.S. \\
\hline 60 & $30.00+3.68$ & $85.83 \pm 6.52$ & $61.67 \pm 4.58$ & H.S. & H.S. & H.S. \\
\hline 80 & 38.83 土 3.49 & $64.17 \pm 5.28$ & $84.17 \pm 2.71$ & H.S. & H.S. & H.S. \\
\hline 100 & $29.83 \pm 2.50$ & $49.17 \pm 3.20$ & $63.33 \pm 7.92$ & H.S. & H.S. & N.S. \\
\hline 120 & $15.67 \pm 1.44$ & $23.17 \pm 3.91$ & $36.33 \pm 6.34$ & N.S. & H.S. & $\mathrm{S}$ \\
\hline 140 & $9.50 \pm 0.63$ & $13.83 \pm 2.26$ & $20.33 \pm 3.15$ & N.S. & H.S. & $\mathrm{S}$ \\
\hline 420 & $11.75 \pm 0.69$ & $16.17 \pm 1.81$ & $16.17 \pm 1.70$ & $\mathrm{~S}$ & S & N.S. \\
\hline 440 & $28.92 \pm 3.08$ & $50.17 \pm 3.63$ & $54.33 \pm 3.48$ & H.S. & H.S. & N.S. \\
\hline 460 & $31.83 \pm 2.68$ & $47.00 \pm \mathbf{3 . 8 7}$ & $76.83 \pm 5.04$ & H.S. & H.S. & H.S. \\
\hline 480 & $17.92 \pm 1.63$ & $17.17 \pm 2.14$ & $39.17 \pm 4.71$ & N.S. & H.S. & H.S. \\
\hline 500 & $13.00 \pm 0.86$ & $15.67 \pm 1.14$ & $17.00 \pm 3.08$ & N.S. & N.S. & N.S. \\
\hline
\end{tabular}

a See note on Table 1

for these times were $40 \mathrm{~min}$ for glipizide and $63 \mathrm{~min}$ for glibenclamide (51 min for controls).

In the second experimental phase, i.e., after $7 \mathrm{~h}$, insulin secretion was greater in the group treated with glibenclamide; this is evident comparing the levels from the 460th min onwards, the peaks and the areas, which weré all highly significantly greater than controls and the glipizide group. to the stimulus, and a marked increase was noted in insulin secretion. At 5 min glipizide-induced secretion was significantly greater than that provoked by glibenclamide.

Insulin secretion remained high throughout the stimulus for both products; during this period no significant differences were noted.

When the drugs were withdrawn from the perfusing 
solution, a marked difference in behaviour was noted between the glipizide and glibenclamide treated pancreas. After glipizide perfusion insulin secretion returned rapidly to basal values, reaching them at the 35 th min.

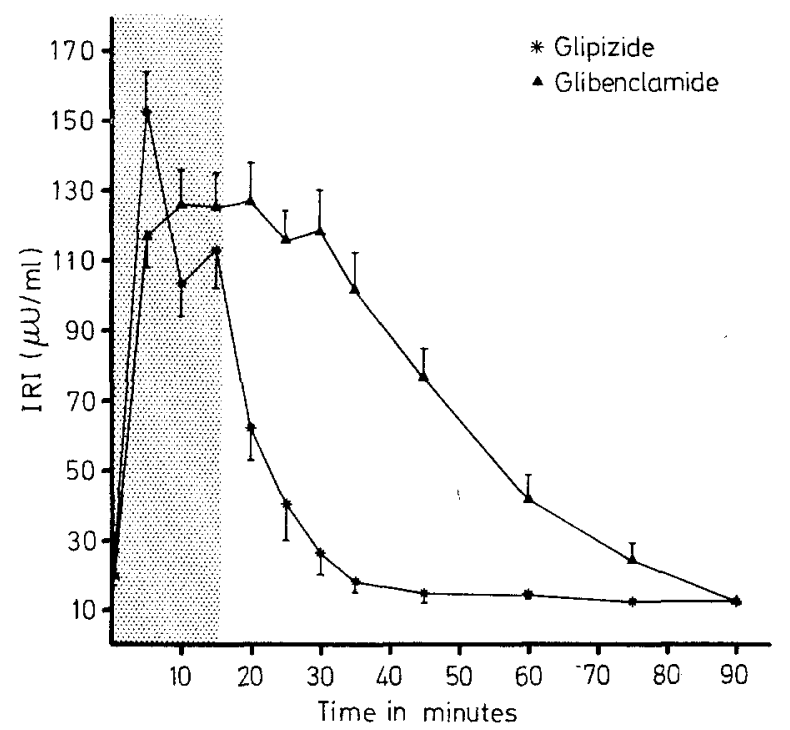

Fig. 5. Effect of glipizide and glibenclamide on insulin secretion by the isolated rat pancreas, perfused from time 0 to the 15th min. Except for times over $45 \mathrm{~min}$, which were obtained with 9 replications, each point represents the mean of 11 replications

After glibenclamide perfusion insulin secretion remained at the levels reached while the product was being perfused until the $30 \mathrm{th}$ min, then started to decrease slowly, reaching basal values only after $90 \mathrm{~min}$. From the 20th to the 75th min the differences between the means for the two treatments were highly significant.

\section{Discussion}

Comparison of glipizide and glibenclamide in the dog with no glucose load has shown that the two hypoglycaemic agents differ markedly. Glipizide acts rapidly, with a prompt hypoglycaemic effect, corresponding to an early insulin secretion peak which returns rapidly to basal levels. With glibenclamide the hypoglycaemic effect becomes evident only after a latent period, and the insulin response is of longer duration.

In the present experiment, the products were administered orally as they are used in therapy. Fuccella et al. [5] demonstrated that in man glipizide is absorbed faster than glibenclamide; this finding might suggest that the difference in the two products' pharmacodynamics was due only to different pharmacokinetics. The differences we observed, however, are similar to those reported by Loubatières et al. [12] Loubatières [13], Raptis et al. [14], and by Haupt et al. [15] between glibenclamide on the one hand, and tolbutamide, glibornuride and glisoxepide on the other, injecting the products intravenously in man. The results of this experiment therefore suggest that, pharmacokinetic characteristics apart, glibenclamide shows different pharmacodynamics from other sulphonylurea group drugs, while glipizide resembles them:

The experiment using two glucose loads in the dog, particularly the results referring to the first load, confirm the speed of action of glipizide, which completely inhibits the glucose-induced hyperglycaemia, whereas glibenclamide acts after a lag-time and in fact only partially antagonizes the hyperglycaemia.

The delay in glibenclamide's action is also seen in plasma insulin, although surprisingly, the insulin levels after glibenclamide treatment are not markedly different from those following glipizide, despite the former being only partially able to lower plasma glucose.

The plasma insulin pattern after the first glucose load also does not differ between glipizide and glibenclamide, contrary to the findings in the previous experiment on animals that had not received glucose. Similar results were obtained by Raptis et al. [14] in experiments comparing glibenclamide and tolbutamide.

Immediately before the second glucose load, the plasma glucose in glibenclamide-treated animals was significantly lower than in controls and the glipizide group. This might appear to suggest that glibenclamide shows more persistent action, even if not mediated by greater and longer-lasting insulin secretion; the results regarding the plasma glucose levels after the second glucose load, however, do not favour this hypothesis, since glibenclamide antagonizes the rise less than glipizide, even though plasma insulin levels were much higher and longer-lasting than in both control and glipizide groups.

That the insulin secretion dynamics following glibenclamide differ from those following glipizide, is confirmed by the results of our experiment on the perfused pancreas. Here the difference was easily seen, and agrees with the findings of Fussgänger et al. [16], Grodsky et al. [17] and Pfeiffer et al. [18] on comparison of glibenclamide with other known sulphonylureas. All these data show that the action of glibenclamide is less intense to start with, but is long-lasting.

These findings may provide at least a partial explanation of different pharmacodynamies of the two hypoglycaemic agents.

\section{References}

1. Ambrogi, V., Bloch, K., Daturi, S., Griggi, P., Logemann, W., Parenti, M.A., Rabini, T., Tommasini, R.: New oral antidiabetic drugs. Part I. ArzneimittelForsch. 21, 200-204 (1971)

2. Ambrogi, V., Bloch, K., Cozzi, P., Daturi, S., Logemann, W., Parenti, M.A., Tommasini, R.: New oral 
antidiabetic drugs. Part II. Arzneimittel-Forsch. 21, $204-208$ (1971)

3. Ambrogi, V., Bloch, K., Daturi, S., Logemann, W. Parenti, M. A., Tommasini, R.: New oral antidiabetic drugs. Part III. Arzneimittel-Forsch. 22, 542-544 (1972)

4. Ambrogi, V., Bloch, K., Daturi, S., Griggi, P., Logemann, W., Mandelli, V., Parenti, M.A., Rabini, T., Usardi, M.M., Tommasini, R.: Pharmacological study on a new oral antidiabetic: $N-\{4-[\beta-(5-$ methyl-pyrazine - 2 - carboxamido) - ethyl] - benzenesulphonyl $\}-N^{\prime}$ cyclohexyl-urea or K 4024. Arzneimittel-Forsch. 21, $208-215$ (1971)

5. Fuccella, L. M., Tamassia, V., Valzelli, G.: Metabolism and kinetics of the hypoglycaemic agent glipizide in man. Comparison with glibenclamide. Clin. Pharm. and New Drugs. (In press)

6. Valzelli, G., Tamassia, V.: Personal communication

7. Frings, C.S., Ratliff, C.R., Dunn, R.T.: Automated determination of glucose in serum or plasma by direct o-toluidine procedure. Clin. Chem. 16, 282-284 (1970)

8. Hales, C.N., Randle, P.J.: Immuno-assay of insulin with insulin-antibody precipitate. Biochem. J. 88, $137-146(1963)$

9. Kramer, C.Y.: Extention of multiple range test to group means with unequal number of replications. Biometrics 12, $307-310$ (1956)

10. Loubatières, A., Mariani, M.A., Ribes, G., de Malbosc, H., Chapal, J.: Etude expérimentale d'un nouveau sulfamide hypoglycémiant particulièrement actif, le HB 419 ou glibenclamide. Diabetologia $5,1-10$ (1969)

11. Wilcoxon, F.: Individual comparison by ranking methods. Biomet. Bull. 1, 80-83 (1945)

12. Loubatières A., Mariani, M.M.: Etude pharmacologique et pharmacodynamique d'un sulfonylurée hypoglycémiant particulièrement actif, le glybenzcyclamide. C.R. Acad. Sci. (Paris) 265, 643-645 (1967)

13. Loubatières, A.: Stimulators and inhibitors of insulin secretion. Physiological and pharmacological inter- ferences synergisms and antagonisms. In: Mechanism and regulation of insulin secretion, p. 243-244. Levine, R., Pfeiffer, E. F. (Eds.). Milano: Casa Editrice "Il Ponte", 1968

14. Raptis, S., Rav, R.M., Schröder, K.E., Faulchaber, D.J., Pfeiffer, E.F.: Comparative study of insulin secretion following repeated administration of glucose, tolbutamide and glibenclamide (HB 419) in diabetic and non-diabetic human subjects. Horm. Metab. Res. 1 suppl., $65-72(1969)$

15. Haupt, E., Köberich, W., Beyer, J., Schöffling, K.: Pharmacodynamic aspects of tolbutamide, glibenclamide, glibornuride and glisoxepide. I) Dose response relations and repeated administration in diabetic subjects. II) Repeated administration in combination with glucose. Diabetologia 7, 449-460 (1971)

16. Fussgänger, R.D., Goberna, R., Hinz, M., Jaros, P., Karsten, C., Pfeiffer, E.F.: Comparative studies on the dynamics of insulin secretion following HB 419 and tolbutamide on the perfused isolated rat pancreas on the perifused isolated islets of Langerhans. Horm. Metab. Res. 1 suppl. 34-40 (1969)

17. Grodsky, G.M., Lee, J., Fanska, R., Smith, D.: Insulin secretion from the "in vitro" perfused pancreas of the rat. Effect of RO 6-4563 and other sulfonylureas. Recent Hypoglycemic Sulfonylureas, pp. 83-96. Bern: Hans Huber 1971

18. Pfeiffer, E. F., Fussgänger, R., Hinz, H., Kastilambros, N., Laube, H.: Dynamics of insulin and glucagon secretion of the isolated perfused pancreas and islets following and in presence of newer sulfonylureas. Recent Hypoglycemic Sulfonylureas. Bern: Hans Huber 97-112 (1971)

Dr. M. A. Parenti

Istituto Carlo Erba

Per Ricerche Terapeutiche

Via C., Imbonati, 24

I-20159 Milano

Italy 